\title{
Respiratory and Systemic Effects of Inhaled Dexamethasone on Ventilator Dependant Preterm Infants at Risk for Bronchopulmonary Dysplasia
}

\author{
Marianne Pappagallo, Soraya Abbasi and Vinod K. Bhutan \\ Section on Newborn Pediatrics, Pennsylvania Hospital, 800 Spruce Street, Philadelphia
}

\begin{abstract}
Short term inhaled dexamethasone therapy was evaluated in a double blind placebo controlled trial in 36 ventilator dependent preterm neonates $(B W<1500 \mathrm{gm}$, postnatal age $>7$ days) who were at risk for bronchopulmonary dysplasia. Pulmonary and systemic effects were compared at early (day 3), late (7-10 days) and post (14 days after initiation) phases of therapy. Airflow mechanics improved as demonstrated by a net $101 \%$ improvement in pulmonary resistance (a decrease from 139 to $101 \mathrm{~cm} \mathrm{H} / 2 \mathrm{OL} / \mathrm{s}$ in the dexamethasone treated infants as compared to an increase from 153 to $267 \mathrm{cmH}_{2} \mathrm{O} / \mathrm{L} / \mathrm{s}$ in the placebo treated infants during the early phase of therapy); this was associated with a $45 \%$ increase in inspiratory airflow $(1.29 \pm 0.43$ to $1.87 \pm 0.978$ $\mathrm{L} / \mathrm{min} ; \mathrm{p}<0.01$ ), and $37 \%$ increase in expiratory airflow. These changes resulted in a significant reduction in the work of breathing such that the mean tidal driving pressure significantly decreased from $13.6 \mathrm{cmH}_{2} \mathrm{O}$ to $9.4 \mathrm{~cm} \mathrm{H} \mathrm{H}_{2} \mathrm{O}$ with inhaled steroid administration. Though the brief duration of therapy did not result in cessation of ventilatory support, the level of support was significantly reduced (decreased values of oxygen supplementation, mean airway pressure and oxygenation index and increased ventilatory efficiency index). The inhaled dexamethasone therapy was also associated with systemic absorption of the drug as evidenced by transient but apparently reversible reduction in serum cortisol levels. No systemic side effects of hypertension, hyperglycemia or nosocomial sepsis were observed. These data demonstrate beneficial effects of short term inhaled dexamethasone on the resistive airflow properties of preterm infants at risk for BPD and may provide adjunctive means to facilitate weaning in the ventilator dependent neonates. (Indian J Pediatr 1998; 65 : 273-282)
\end{abstract}

Key words : Inhalation; Dexamethasone; Bronchopulmonary dysplasia

Postnatal glucocorticoid therapy has been resurging with the hope of improving the respiratory status of the ventilator dependent preterm neonates recovering from respiratory distress syndrome (RDS). Several clinical reports and clinical trials have pre-

Reprint requests : Soraya Abbasi, M.D., Section on newborn Pediatrics Pennsylvania Hospital, 800 Spruce Street, Philadelphia, Pennsylvania 19107. viously suggested the beneficial use of steroids for bronchopulmonary dysplasia (BPD) defined by oxgyen dependancy at 4 weeks of age or ventilator dependency at 2 weeks of age. ${ }^{1,2,3}$ In these instances, reports have shown improvements in pulmonary mechanics, gas exchange and facilitation in weaning from ventilatory support. The use of postnatal steroids have also been suggested within the first three days of life to reduce the severity of RDS. ${ }^{4}$ The most fre- 
quent route of glucocorticoid administration has been systemic, though its duration has varied at different centers. $5,6, \overline{7}$ Prolonged systemic usage ( $>3$ weeks) has been associated with hypertension, hyperglycemia, adrenal suppression and the possibility of an increased incidence of sepsis; somatic growth retardation has been considered but not proven. ${ }^{3}$ Inhalational mode of glucocorticoid administration has been suggested by some investigators and more recently, LaForce and Brudno have reported an improvement in pulmonary compliance and resistance in a controlled trial of 4 weeks treatment with nebulized beclomethasone dipropionate in ventilator dependent infants at 2 weeks of age. ${ }^{8}$

Our study evaluates the pulmonary and systemic effects of nebulized dexamethasone in a placebo controlled randomized trial in ventilated preterm neonates (< $1500 \mathrm{~g}$ birth weight) of $>7$ days of age who were predicted to develop BPD, and in those between 2 to 4 weeks of age who had failed to wean with standard techniques and were likely to develop BPD. We specifically selected a shorter duration of treatment ( 10 days) to assess the changes in the level of ventilatory support and pulmonary and airflow function.

\section{MATERIALS AND METHODS}

This study was conducted in the intensive care nursery at Pennsylvania Hospital and was reviewed and approved by the Institutional Review Board. Study patients of less than $1500 \mathrm{~g}$ birth weight were enrolled with informed parental consent. The neonates selected for the study were evaluated in two study phases. The first phase was the pilot study of 9 neonates who were individually matched to a group of controlled infants by birth weight, gestational age and PNA. The mean birthweight was $915 \mathrm{gm}$ and mean gestational age was 26.5 weeks. The second phase was the double blind placebo-controlled randomized study of 18 neonates with similar inclusion criteria. Infants were considered eligible if they were : (i) older than 7 days of age and predicted to have more than $75 \%$ probability of BPD based upon the previously described prediction model ${ }^{9}$, (ii) older than 2 weeks of age and predicted to develop BPD based on the BPD predictive model, and (iii) being ventilator dependent had failed stand. ard weaning techniques and had abnormal pulmonary funtions. "Pre BPD" babies were administered methylxanthine therapy. Those over 2 weeks of age were administered diuretics. None of these therapies were instituted or altered during the study period. Infants with congenital anomalies, congenital heart disease, pulmonary hypoplasia, and sepsis were excluded.

\section{Dexamethasone Inhalation}

Injectable dexamethasone preparation was used for inhalational purposes. Using a protocol developed by our pharmacist, $0.4 \mathrm{mg} / \mathrm{ml}$ of dexamethasone was prepared in $1.0 \mathrm{ml}$ vials. The dosage for the pilot study was $0.5 \mathrm{mg} / \mathrm{kg} /$ day from days 0 to 3 and was reduced to $0.25 \mathrm{mg} / \mathrm{kg} /$ day from days 4 to 7 . During the second phase, the dosage and duration was increased to $1.0 \mathrm{mg} / \mathrm{kg} /$ day from days 0 to 7 and then reduced to $0.5 \mathrm{mg} / \mathrm{kg} /$ day from days 8 to 10. The calculated amount of dosage was diluted in 2 cc normal saline, and administered with a jet nebulizer over a 20 to 30 minute period every 8 hours during the study period. For intubated neonates, the nebulizer was connected to the inspiratory 
port of the ventilator circuit closest to the endotracheal adapter. Infants who were extubated prior to the completion of the study continued to receive the therapy by face mask nebulization.

Placebo was normal saline administered in a similar volume and manner. The $10 \mathrm{ml}$ vials prepared by the pharmacist were labelled with a code for a double blind study. Since both study and placebo medications were clear solutions and dosage calculated on the basis of volume, the ICN staff remained unaware of the type of medication.

Ventilatory support and respiratory management were provided by the ICN staff in their usual manner. Blood gases were monitored by indwelling arterial lines or by non-invasive techniques of pulse oximetry and transcutaneous $\mathrm{CO}_{2}$ monitoring. The magnitude of respiratory support was gauged by calculating (i) the oxygenation index (OI) where, $\mathrm{OI}=\mathrm{MAP} \times \mathrm{FiO}_{2} / \mathrm{PaO}_{2}$ and (ii) the ventilator efficiency index (VEI) where, VEI $=5 /($ PIP-PEEP $) \times$ IMV $\times$ $\left(\mathrm{PaCO}_{2} / 760\right)$. Additional data evaluated were systemic blood pressure (doppler technique), serum glucose, and evidence of sepsis. Serum cortisol was measured in samples collected between 7 and 8 AM (to standardize for diural fluctuations) prior to onset of therapy and on days 7 and 24 of the study period.

\section{Pulmonary Function Measurement}

The pulmonary functions were measured prior to the initiation of therapy and repeated at days 3 (early), 7 and 10 (late) of the therapy and post therapy (at 14 days after initiation of study). Each evaluation was done for the infant in a non-sedated, supine, head neutral posture. Oropharyngeal and tracheal suction were done 30 to
35 minutes prior to the study and the studies were done 2 hours beyond the last feed for those on enteral nutrition. Using the methodology described previously, pulmonary functions were measured by the simultaneous determinations of airflow and transpulmonary pressure by the use of a pneumotachometer and the esophageal balloon technique ${ }^{10}$. Data were obtained for 60 to 100 seconds to sample and analyze 25 to 60 breaths, and the mean values of the collected data were used to provide measured values of tidal volume, minute ventilation, inspiratory/expiratory airflow and peak to peak esophageal pressure (driving pressure). Calculated values of pulmonary functions, dynamic compliance and pulmonary resistances for the total and inspiratory/expiratory phases of the respiratory cycles were obtained by the least mean squares analysis technique. ${ }^{10}$ All data were measured during spontaneous breathing.

\section{Statistical Analysis}

The initial pilot study was conducted with age/weight matched controls to both ascertain the technical feasibility of using dexamethasone as an inhalational agent; and determine if any effect on pulmonary mechanics was demonstrable. Using the experience and data of the pilot study, the second phase of the study was designed to examine the pulmonary airflow and systemic effects of inhaled dexamethasone. Based upon earlier experience, a decision was made to use a larger dose and longer duration of dexamethasone. Using the data from the pilot study ${ }^{11}$ and literature ${ }^{9}$, a $35 \%$ improvement in pulmonary function parameters was deemed appropriate. Power analysis determined a sample size of 9 
for each arm of the study. The statistical design of the study was based on a double blinded prospective randomized analysis. Data were analyzed with SAS/STAT Software (SAS Institute, Cary, NC). Comparisons were made using student $t$-test and repeated measure analysis of variance (ANOVA); $\mathrm{p}$ values $<0.05$ were considered significant.

\section{RESULTS}

A total of 36 neonates were studied. Dur- ing the first phase of the study, changes in pulmonary mechanics were measured (Table 1). Significant improvements in pulmonary compliance and total pulmonary resistance were observed with the inhaled dexamethasone therapy. Extubation was not facilitated and the effect on weaning or reduction of ventilatory support was not clearly or consistently evident. The demographic and the overall ICN course of the eighteen neonates assigned to the second phase of the study are shown in Table 2 . There were no statistical differences be-

TABle 1. Pulmonary Function Data in Phase I of Inhaled Steroid $(0.5 \mathrm{mg} / \mathrm{kg} /$ day $)$

\begin{tabular}{|c|c|c|c|c|}
\hline \multirow{2}{*}{ Pulmonary function } & \multicolumn{2}{|c|}{ Pre therapy } & \multicolumn{2}{|c|}{ Post 7 days therapy } \\
\hline & $\begin{array}{l}\text { No dexamethasone } \\
\qquad(\mathrm{n}=9)\end{array}$ & $\begin{array}{l}\text { Dexamethasone } \\
\qquad(\mathrm{n}=9)\end{array}$ & $\begin{array}{l}\text { No dexamethasone } \\
\qquad(\mathrm{n}=9)\end{array}$ & $\begin{array}{l}\text { Dexamethasone } \\
\quad(\mathrm{n}=9)\end{array}$ \\
\hline Tidal volume $(\mathrm{mg} / \mathrm{kg})$ & $5.70 \pm 0.35$ & $6.22 \pm 0.54$ & $5.75 \pm 0.53$ & $5.67 \pm 0.44$ \\
\hline $\begin{array}{l}\text { Minute ventilation } \\
(\mathrm{ml} / \mathrm{min} / \mathrm{kg})\end{array}$ & $408.0 \pm 28.7$ & $428.0 \pm 31.8$ & $383.5 \pm 30.2$ & $402.8 \pm 29.1$ \\
\hline $\begin{array}{l}\text { Compliance } \\
(\mathrm{ml} / \mathrm{cmH} 20 / \mathrm{kg})\end{array}$ & $0.71 \pm 0.08$ & $0.46 \pm 0.06$ & $0.96 \pm 0.13$ & $0.74 \pm 0.10$ \\
\hline $\begin{array}{l}\text { Resistance } \\
\qquad\left(\mathrm{cmH}_{2} \mathrm{O} / \mathrm{L} / \mathrm{s}\right)\end{array}$ & $88.6 \pm 14.8$ & $121.1 \pm 18.1$ & $70.5 \pm 10.6$ & $91.9 \pm 141.2$ \\
\hline
\end{tabular}

Data are mean \pm SEM ; dexamethasone treated babies compared to age and weight matched controls do not show significant changes

TABLE 2. Demographic Data Phase II of Inhaled Steroid Study $(1.0 \mathrm{mg} / \mathrm{kg} / \mathrm{dose})$

\begin{tabular}{lcr}
\hline & $\begin{array}{c}\text { Dexamethasone } \\
(\mathrm{n}=9)\end{array}$ & \multicolumn{1}{c}{$\begin{array}{c}\text { Placebo } \\
(\mathrm{n}=9)\end{array}$} \\
\hline Birthweight $(\mathrm{g})$ & $828 \pm 64$ & $849 \pm 89$ \\
Study weight $(\mathrm{g})$ & $866 \pm 62$ & $845 \pm 72$ \\
Gestational age (weeks) & $26.8 \pm 1.1$ & $26.6 \pm 0.8$ \\
Male to female & $5: 4$ & $5: 4$ \\
Days on ventilator & $62.6 \pm 10.3$ & $58.6 \pm 8.9$ \\
Days on oxygen & $91.4 \pm 15.8$ & $88.3 \pm 17.9$ \\
Age study began (days) & $22.6 \pm 3.0$ & $19.13 \pm 1.6$ \\
Age study ended (days) & $32.5 \pm 3.0$ & $27.8 \pm 1.6$ \\
Age at discharge (days) & $100.0 \pm 20.3$ & $124.7 \pm 16.4$ \\
\hline
\end{tabular}



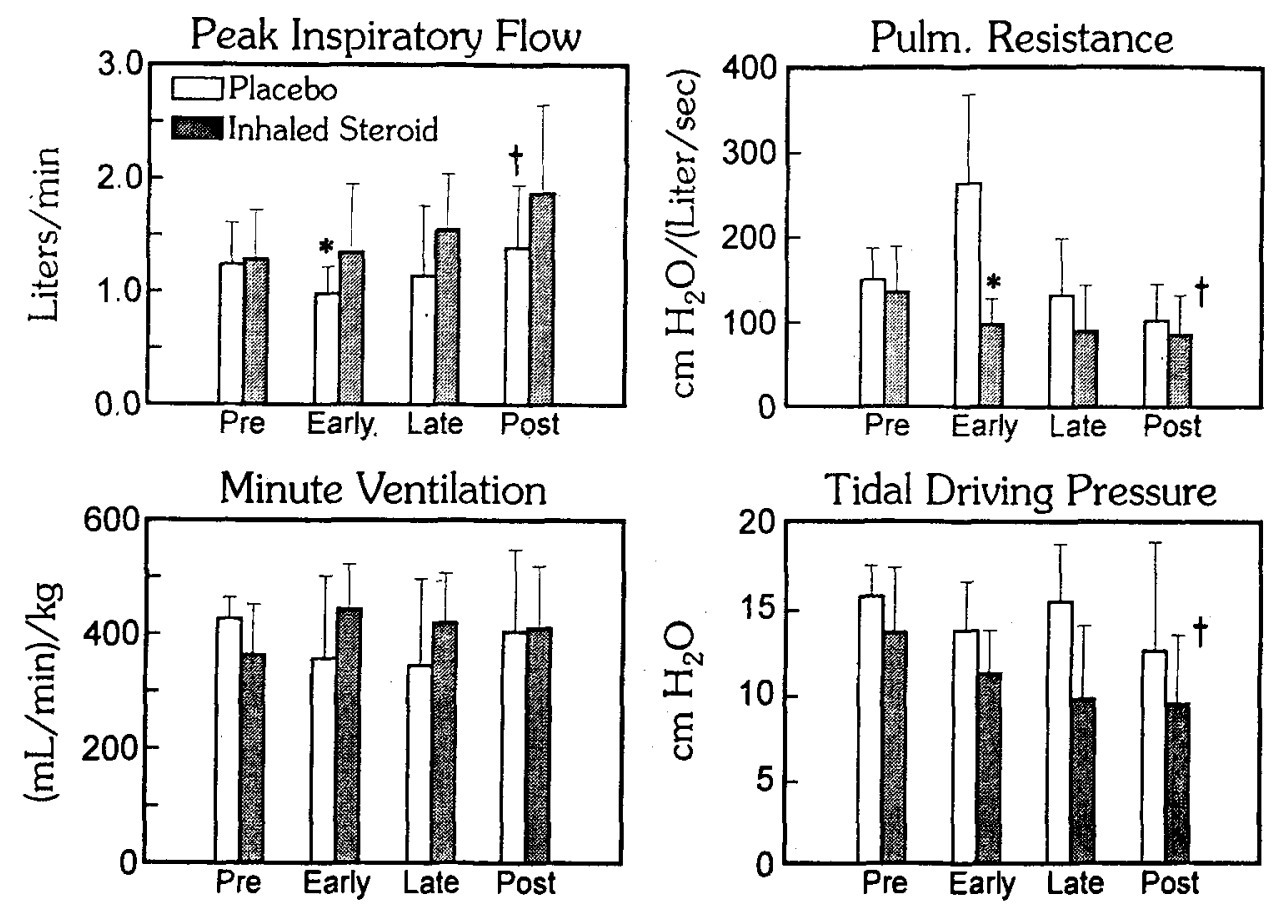

rig. 1. Effect of inhaled dexamethasone therapy (shaded bars) as compared to placebo (open bars) on airflow and pulmonary function parameters during early (day 1 to 3), late (day 7 to 10) phases of treatment and 3 days after its discontinuation (post). ${ }^{*} p<0.01$ by ' $t$ ' test and $p<$ 0.05 by ANOVA. No significant changes were observed in the placebo group over the duration of the study.

tween the study or the placebo group for birth weight, gestational age, duration of ventilatory support, oxygen administration or duration of stay in the hospital.

Effect on airflow and pulmonary function parameters for the second phase of the study are illustrated in Fig. 1. The total pulmonary resistance improved significantly immediately after initiation of dexamethasone. During the early phase of the dexamethasone treatment, the pulmonary resistance value decreased by $27 \%$ from a mean value $139 \pm 54 \mathrm{SD} \mathrm{cmH} \mathrm{C}_{2} \mathrm{O} / \mathrm{L} / \mathrm{s}$ to $101 \pm 29$ $\mathrm{SD} \mathrm{cmH}_{2} \mathrm{O} / \mathrm{L} / \mathrm{s}$ while in the placebo group these values increased by $74 \%$ from $153 \pm$ $37 \mathrm{SD} \mathrm{cmH}_{2} \mathrm{O} / \mathrm{L} / \mathrm{s}$ to $267 \pm 104 \mathrm{SD}$ $\mathrm{cmH}_{2} \mathrm{O} / \mathrm{L} / \mathrm{s}(\mathrm{p}<0.01)$. Fourteen days after the initiation of a ten day therapy, the inspiratory airflow remained significantly improved in the dexamethasone group. These values were $45 \%$ higher from a mean pre-study value $1.29 \pm 0.43 \mathrm{SD} \mathrm{L} / \mathrm{min}$ to a value of $1.87 \pm 0.78 \mathrm{SD} \mathrm{L} / \mathrm{min}$, $(\mathrm{p}<0.05)$ as compared to only a $12 \%$ increase in the placebo group during the same study period $(1.27 \pm 0.37$ to $1.39 \pm 0.55 \mathrm{SD} \mathrm{L} / \mathrm{min})$.

Minute ventilation during spontaneous breathing and values of dynamic compli- 
TABLE 3. Level of Ventilatory Support for Phase II of Inhaled Steroid Study

\begin{tabular}{|c|c|c|c|c|c|}
\hline \multicolumn{2}{|c|}{ Ventilatory parameters } & \multicolumn{4}{|c|}{ Phases of inhaled therapy } \\
\hline & & Pre & Early & Late & Post \\
\hline \multirow{5}{*}{$\begin{array}{l}\text { Placebo } \\
(n=9)\end{array}$} & $\mathrm{FiO}_{2}$ & $0.52 \pm 0.08$ & $0.61 \pm 0.08$ & $0.43 \pm 0.05$ & $0.53 \pm 0.05$ \\
\hline & $\mathrm{MAP}\left(\mathrm{cmH}_{2} \mathrm{O}\right)$ & $7.00 \pm 0.46$ & $7.88 \pm 0.74$ & $7.50 \pm 0.65$ & $7.57 \pm 0.78$ \\
\hline & $\mathrm{IMV}(\mathrm{br} / \mathrm{min})$ & $40.50 \pm 1.92$ & $39.75 \pm 3.76$ & $34.50 \pm 3.86$ & $35.71 \pm 4.25$ \\
\hline & OI & $0.06 \pm 0.01$ & $0.09 \pm 0.01$ & $0.07 \pm 0.01$ & $0.08 \pm 0.01$ \\
\hline & VEI & $0.17 \pm 0.04$ & $0.17 \pm 0.07$ & $0.21 \pm 0.12$ & $0.23 \pm 0.15$ \\
\hline \multirow{5}{*}{$\begin{array}{l}\text { Dexamethasone } \\
(n=9)\end{array}$} & $\mathrm{FiO}_{2}$ & $0.55 \pm 0.08$ & $0.47 \pm 0.05$ & $0.39 \pm 0.05$ & $0.38 \pm 4.0$ \\
\hline & $\operatorname{MAP}\left(\mathrm{cmH}_{2} \mathrm{O}\right)$ & $7.89 \pm 0.54$ & $6.22 \pm 0.55$ & $5.11 \pm 0.51^{*}$ & $5.29 \pm 0.29$ \\
\hline & IMV (br/min) & $0.22 \pm 5.53$ & $28.00 \pm 3.37$ & $21.44 \pm 3.52^{*}$ & $25.43 \pm 3.65$ \\
\hline & OI & $0.09 \pm 0.01$ & $0.05 \pm 0.005$ & $0.04 \pm 0.005^{*}$ & $0.04 \pm 0.008^{*}$ \\
\hline & VEI & $0.26 \pm 0.02$ & $0.32 \pm 0.02^{*}$ & $0.49 \pm 0.12^{*}$ & $0.37 \pm 0.12^{*}$ \\
\hline
\end{tabular}

$\mathrm{IMV}=$ Intermittent Mandatory Ventilation; $\mathrm{MAP}=$ Mean Airway Pressure; $\mathrm{FiO}_{2}=$ Fraction of Inspired Oxygen; OI = Oxygenation Index; and VEI = Ventilatory Efficiency Index. Data are mean \pm $\mathrm{SD}$, level of significance $\left({ }^{*}\right)$, $\mathrm{p}$ values $<0.05$ for dexamethasone treated group compared to placebo group (ANOVA and Students ' $t$ ' tests).

ance were not significantly altered by the inhaled dexamethasone during the 10 day therapy. However, the driving pressure to achieve the tidal breath was significantly reduced both during the early and late phases of the therapy $(p<0.05)$. The mean values of driving pressure reduced by $37 \%$ from values of $13.6 \pm 3.8 \mathrm{SD} \mathrm{cmH}_{2} \mathrm{O}$ pre study to $9.4 \pm 4.0 \mathrm{~cm} \mathrm{H}_{2} \mathrm{O}$ at the end of the therapy. Similar changes were observed with resistive work of breathing.

Concomitant changes in the level of ventilatory support and oxygen administration are described by the $\mathrm{FiO}_{2}$, mean airway pressure, ventilatory efficiency index, and intermittent mandatory ventilation rate at days 3,7 and 14 of initiation of therapy (Table 3). Significant reductions in mean airway pressure (MAP), oxygenation index (OI), fractional inspiratory oxygen $\left(\mathrm{FiO}_{2}\right)$ and intermittent mandatory ventila- tion (IMV) were evident by 7 days of dexamethasone therapy and persisted upon completion of therapy when compared to placebo. The ventilatory efficiency indices were also significantly improved over the duration of the therapy as compared to placebo.

Data on systemic blood pressure and cortisol are shown in Table 4. There was no statistical evidence of either systolic or diastolic hypertension. The serum cortisol levels were significantly lower at day 7 upon dexamethasone therapy; no differences were observed upon completion of therapy. There was no difference in the incidence of hyperglycemia or nosocomial sepsis during one week post therapy for the two groups. No differences in blood gas tension values were demonstrated.

During the study period, the incidence of successful extubation was not remarka- 
TABle 4. Blood Pressure and Serum Cortisol Data in Phase II of Inhaled Steroid Study $(1.0 \mathrm{mg} / \mathrm{kg} /$ day)

\begin{tabular}{|c|c|c|c|c|c|}
\hline \multicolumn{2}{|c|}{ Parameter } & \multicolumn{4}{|c|}{ Phases of inhaled therapy } \\
\hline & & Pre & Early & Late & Post \\
\hline $\begin{array}{l}\text { Blood pressure } \\
\text { Systolic/Diastolic }\end{array}$ & $\begin{array}{r}\text { Placebo } \\
(n=9)\end{array}$ & $62 / 34$ & $68 / 36$ & $63 / 37$ & $64 / 36$ \\
\hline \multicolumn{2}{|c|}{$\begin{array}{r}\text { Dexamethasone } \\
(\mathrm{n}=9)\end{array}$} & $65 / 35$ & $66 / 38$ & $68 / 38$ & $64 / 34$ \\
\hline \multirow{2}{*}{$\begin{array}{l}\text { Serum cortisol + } \\
\text { (IU/100 ml) }\end{array}$} & $\begin{array}{r}\text { Placebn } \\
(n=9)\end{array}$ & $10.5 \pm 3.1$ & -- & $13.3 \pm 6.3$ & $7.88 \pm 2.6$ \\
\hline & $\begin{array}{c}\text { ethasone } \\
(\mathrm{n}=9)\end{array}$ & $10.6 \pm 2.2$ & - & $2.7 \pm 1.1^{*}$ & $4.94 \pm 3.2$ \\
\hline
\end{tabular}

Blood pressure data is presented as mean vaues. + Serum cortisol data are presented as mean \pm $\mathrm{SD}$; level of significance $\left(^{*}\right), \mathrm{P}<0.05$

bly different. Systemic steroids were administered in $4 / 9$ infants within a week of study completion in the placebo group as compared to $2 / 9$ infants in the inhaled dexamethasone group.

\section{DISCUSSION}

Inhalational steroids administered to preterm neonates at risk for BPD for a duration of 7 to 10 days improve their resistiveairflow function and facilitate their weaning from ventilatory assistance. Our data demonstrates a net $101 \%$ improvement in pulmonary resistance, a $45 \%$ increase in inspiratory airflow, a $37 \%$ increase in expiratory airflow and a net $37 \%$ reduction in driving pressure for each tidal breath. Thus, the mean tidal driving pressure was reduced from $13.6 \pm 3.8 \mathrm{cmH}_{2} \mathrm{O}$ to $9.4 \pm 4.0$ $\mathrm{cmH}_{2} \mathrm{O}$. Though the brief duration of therapy did not result in cessation of ventilatory support, the level of support was significantly decreased and thus weaning from support could have been facilitated. These data are similar to the improvements previ- ously observed with 4 weeks of inhalational steroids initiated at 2 weeks of age ${ }^{8}$.

The role of systemic corticosteroid therapy for BPD has been evaluated by several controlled clinical trials ${ }^{3-7,12}$. These have confirmed the beneficial responses by reduction in the need for supplemental inspired oxygen and ventilatory support as well as an improvement in pulmonary compliance and facilitation of extubation. The age of initiation (1 to 4 weeks of age) and the duration of therapies (2 to 8 weeks) have generally varied and are currently guided by the Colloborative Dexamethasone Trial Group data ${ }^{3}$ that suggest that the adverse effects of dexamethasone therapy do not appear to outweigh their benefits. However, experience would suggest that prolonged usage of steroids, the early age of therapy initiation, repeat courses of steroid therapy, or susceptibility of the very, very low birthweight neonate who warrants therapy, could place them at an increased risk of steroid related adverse effects. It is in this context that the role of an inhalational routine was conceived to facil- 
itate weaning ventilator dependent neonates $^{11}$.

Previous studies have demonstrated an improvement in pulmonary functions with prolonged therapy ${ }^{1-8}$; other investigators have attributed this effect to enhanced surfactant synthesis, stabilization of lysosomal and cell membrane, reduction of pulmonary edema and a down regulation of the lung inflammation and decrease in the inflammatory response and microvascular permeability seen in BPD. ${ }^{13,14,15}$ Our observations of improvement primarily in resistive airflow functions within 1 to 3 days of therapy suggest an early airway response to steroids. These changes may be attributed to reduction of tracheo-bronchial mucosal edema, enhancement of $\beta$-adrenergic activity, amelioration of any of bronchoprovocation, and/or inhibition of prostag. landin and leukotriene synthesis. In fact, inhaled steroids have been used successfully in the management of asthmatics to decrease bronchial inflammation and the ensuing hyperresponsiveness. On the other hand, preliminary bronchoscopic evaluation of ventilator-dependent preterm neonates has shown an early onset of tracheobronchial mucosal lesions suggestive of necrotizing tracheobronchitis ${ }^{16}$. Early onset of tracheo-bronchial mucosal edema and hyperemia and even obstructive granulation lesions soon after initiation of ventilation have also been reported by our laboratory ${ }^{17}$. Thus, it is quite feasible that corticosteroid therapy may play an adjunctive beneficial role in treating "occult" obstructive tracheo-bronchial mucosal changes.

The clinical improvement observed with aerosol therapy is thus directly related to the ability of steroids to relieve bronchospasm, decrease mucosal edema and liquify bronchial secretions. The magnitude of improvement also depends on the actual area that the drug is in contact with bronchial mucosa. To enhance topical delivery, it is important to consider the mode of delivery, particle size and the physico-chemical properties of the carrier, the temperature of the mist and the dosage of the drug. It is also feasible that in the non-intubated neonate, the steroids may be swallowed and enterally absorbed. So far, there has been little consensus regarding the mode of delivery, dosage or frequency. We initially selected a dosage of $0.5 \mathrm{mg} / \mathrm{kg} /$ day of dexamethasone and only observed a marginal improvement in pulmonary function. Based on these data, we elected to use a dosage of $1.0 \mathrm{mg} / \mathrm{kg} /$ day and standardized the delivery method for each use to ensure accurate dosage and drug delivery. The duration of therapy in our study is 10 days and differs from the 4 weeks used by LaForce and Brudno ${ }^{8}$. Beclomethasone and budenoside have also been used in a similar manner and have shown a similar magnitude of improvement in pulmonary function. The advantages of these two drugs is that they are rapidly degraded and less well absorbed from the lungs and thereby have a more topical effect ${ }^{18}$. Our choice of dexamethasone was based upon its easy availability and familiarity, the ability to adjust specific dosage for the neonates body weight and the choice to use an agent without preservations.

Adrenal suppression with intravenous dexamethasone has been transient. In two separate studies dexamethasone therapy was weaned over a 7 day and 45 day period and resulted in an initial suppression of the hypothalamic-pituitary axis function ${ }^{19,20}$. Eventually, the serum cortisol levels returned to normal either with low dose dexamethasone or its discontinuation. Our 
study also shows evidence of transient adrenal suppression with increasing values of serum cortisol 4 days after discontinuation of therapy, while the placebo treated infants were unaffected. No other systemic side effects of hypertension, hyperglycemia or nosocomial sepsis were observed in our steroid treated group.

In conclusion, we report a direct beneficial effect of inhaled dexamethasone administration on the airflow resistive properties of preterm newborns at risk for BPD. This response is immediate within the first few days of therapy and facilitates weaning from ventilatory support as shown by significant improvements in VEI and reduction in oxygen supplementation and actual reduction in ventilatory parameters. The effect on adrenal suppression was transient and reversible and side effects were negligible. Thus, inhaled steroids can be used to reduce the level of ventilatory support and improve the airway function in preterm neonates at risk for BPD.

\section{ACKNOWLEDGEMENTS}

The authors appreciate the invaluable technical and research assistance of Jean Anne Cieplinski, RN, and Karen Karp, RN and the bioengineering expertise of Mr. Emidio M. Sivieri. The authors also thank Donna Marie Spitz for her secretarial assistance.

\section{REFERENCES}

1. Avery GB, Fletcher AB, Kaplan M, Brudno DS. Controlled trial of dexamethasone in respirator-dependent infants with bronchopulmonary dysplasia. Pediatrics $1985 ; 75: 106-111$.

2. Cummings JJ, $D^{\prime}$ Eugenio DB, Gross SJ. A controlled trial of dexamethasone in preterm infants as high risk for broncho-pul- monary dysplasia. New Engl I Med 1989; $320: 1505-1510$.

3. Collaborative Dexamethasone Trial Group. Dexamethasone therapy in neonatal chronic lung disease : An international placebo-controlled trial. Pediatrics $1991 ; 88: 421-427$.

4. Yeh TF, Torre JA, Rostogi A, Anyebuno MA, Pildes RS. Early postnatal dexamethasone therapy in premature infants with severe respiratory distress syndrome : a double blind controlled study. J Pediatr 1990; 117 : 273-282.

5. Harkavy KL, Scanlon JW, Chowdhry PK, Grylack LJ. Dexamethasone therapy for chronic lung disease in ventilator and oxygen-dependent infants : A controlled trial. J Pediatr 1989; 115 : 979-983.

6. Ohlsson A, Calvert S, Hosking M, Shen. nan A. Randomized controlled trial of dexamethasone treatment in very low birth weight infants with ventilator dependent chronic lung disease. Pediatr Res 1989; 25 : 225A.

7. Mammel MC, Johnson DE, Green TP, Thompson TR. Controlled trial of dexamethasone therapy in infants with broncho-pulmonary dysplasia : Acute effects and 1-year follow-up. Dev Pharmacol Ther $1987 ; 10: 1-11$.

8. LaForce WR, Brudno DS. Controlled trial of beclomethasone dipropionate by nebulization in oxygen and ventilator-dependent infants. J Pediatr 1993; 122 : 285258.

9. Bhutani VK, Abbasi S. Relative likelihood of bronchopúlmonary dysplasia based on pulmonary mechanics measured in preterm neonates during the first week of life. J Peds 1992; $120: 605-613$.

10. Bhutani VK, Abbasi S, Sivieri EM, Fox WW, Shaffer TM. Evaluation of neonatal pulmonary mechanics and energetics: A two factor least mean square analysis. Pediatr Pulmonol 1988; 4 : 150-158.

11. Pappagallo $M$, Blondheim $O$, Bhutani VK, Abbasi S. Effect of inhaled dexamethasone in ventilator dependent preterm in- 
fants. Pediatr Res 1990; $27: 219$ A.

12. Noble-Jamieson CM, Regev R, Silverman $M$. Dexamethasone in neonatal chronic lung disease : Pulmonary effects and intra-cranial complications. Eur J Pediatr 1989; $148:$ 365-367.

13. Williams TJ, Yarwood H. Effect of glucocorticosteroids on microvascular permeability. Am Rev Respir Dis 1990; 141 : S39. 43.

14. Schleimer RP, Freeland HS, Peters SP, Brown KE, Derse CP. An assessment of the effects of glucocorticoids on degranulation, chemotaxis, binding to vascular endothelium and formation of leukotriene $\mathrm{B}_{4}$ by purified human neutrophils. J Pharmacol Exp Ther 1989; 250: 598-605.

15. Groneck P, Reuss D, Gotze-Speer B, Speer CP. Efferts of dexamethasone on chemotactic activity and inflammatory media- tors in tracheobronchial aspirates of preterm infants at risk for chronic lung disease. J Pediatr 1993; 122 : 938-44.

16. Finer NN, Etches PC. Fiberoptic bronchoscopy in the neonate. Pediatr Pulmonol 1989; $7: 116-20$.

17. Abbasi S, Silva W, Gerdes JS, Bhutani VK. Role of dexamethasone therapy for severe tracheobronchial mucosal lesions in mechanically ventilated preterm infants. Ped Res 1993; 33 : 198A.

18. Alkalay AL, Pomerance JJ, Puri AR, et al. Hypothalamic-pituitary-adrenal axis function in very low birth weight infants treated with dexamethasone. Pediatrics 1990; 86 : 204-210.

19. Wilson D, Baldwin R. A randomized placebo controlled trial of effects of dexamethasone on hypothalamic pituitary adrenal axis in preterm infants. J Pediatr 1988; $113: 764-768$ 